\title{
Emerging concepts in the closed-loop spinal cord stimulation system: preliminary results of the Avalon study
}

\author{
Rinoo Vasant Shah ${ }^{1}$, Alan David Kaye ${ }^{1}$, Richard D. Urman ${ }^{2}$ \\ ${ }^{1}$ Department of Anesthesiology, Ochsner LSU Health Sciences Center, Shreveport, Louisiana, USA; ${ }^{2}$ Department of Anesthesiology, Perioperative \\ and Pain Medicine, Brigham and Women's Hospital, Boston, MA, USA. \\ Correspondence to: Richard D. Urman, MD, MBA. Department of Anesthesiology, Perioperative and Pain Medicine, Brigham and Women's Hospital, \\ 75 Francis Street, Boston, MA 02115, USA. Email: urmanr@gmail.com. \\ Provenance and Peer Review: This article was commissioned and reviewed by the Academic Editor Dr. Hao Zhang (Department of Anesthesiology, \\ Rocket Force Characteristic Medical Center of PLA, Beijing, China). \\ Comment on: Russo M, Cousins MJ, Brooker C, et al. Effective relief of pain and associated symptoms with closed-loop spinal cord stimulation \\ system: preliminary results of the Avalon study. Neuromodulation 2018;21:38-47.
}

Submitted Mar 16, 2020. Accepted for publication Apr 23, 2020.

doi: 10.21037/apm-20-642

View this article at: http://dx.doi.org/10.21037/apm-20-642

Invasive nonsurgical techniques have a central role in the management of patients suffering from acute and chronic pain. Common percutaneous pain procedures include trigger point injections, intra-articular injections, spinal injections, nerve blocks, radiofrequency lesioning, epidural adhesiolysis, intradiscal procedures, and minimally invasive surgical spine procedures (1). Historically, neuromodulation and continuous intrathecal analgesia were offered when conventional therapies fail $(2,3)$.

Neurostimulators deliver electrical energy to neuronal and glial structures. The goal is to improve pain and function. The administration of low-intensity electrical currents avoids pharmacological side effects. Neurostimulation has been used for a variety of chronic pain indications including failed back surgery syndrome, complex regional pain syndrome, diabetic neuropathy, intractable angina pectoris, and chronic migraine $(2,3)$. In the United States, the most common clinical role of spinal cord stimulation is for failed back surgery syndrome. In most European countries, the most common role of spinal cord stimulation is for peripheral ischemia.

Technological advances have been seen in recent years in neurostimulation. These build on the science of interrupting pain impulses mediated by $\mathrm{C}$ fibers and A-delta fibers, stimulating larger A-beta fibers, and activating glial tissue. These upgrades have improved a number of important aspects with regard to patient satisfaction. Russo et al., published the preliminary six-month results of a prospective trial of a novel spinal cord spinal stimulation system (1). This study antecedes a more recent study of the same group of patients at the twelve-month mark (4). The study is sponsored by the manufacturer, Saluda Medical and many of the authors disclose their relationships to this manufacturer.

These clinical studies follow earlier proof of concept studies in animal and human volunteers $(5,6)$. Although the exact mechanism of action of pain relief with spinal cord stimulation systems is not fully elucidated, neurophysiology suggests that delivered current can activate sensory fibers in the spinal cord (6). If this current crosses a threshold, a single fiber action potential is created, best measured in the dorsal columns (5). The sum of these single fiber action potentials is known as an evoked compound action potential. The evoked compound action potential has several measurable parameters, of which the amplitude is the most important clinically. As the current increases, more single fibers are recruited and the evoked compound action potential amplitude increases. The evoked compound action potential and single fiber action potentials are thought to emanate from a few low threshold A-beta fibers in the dorsal columns, whereas unpleasant sensations occur with recruitment of the higher threshold A-beta fibers and A-delta fibers $(5,6)$. Conversely, a drop-in fiber recruitment leads to loss of effect.

In traditional tonic spinal cord systems, the patient 
manually adjusts the amplitude to induce comfortable paresthesias. However, with respiration, heart rate, and postural movement, the distance between various electrode contacts and the spinal cord can shift. Depending on this geographic variation, the patient may develop painful stimulation, paresthesias in unwanted locations, or loss of effect. This variability is poorly tolerated and is a reason for patient dissatisfaction up to and including device removal. Parker, et al, demonstrated in volunteers that these sensations correspond to variations in evoked compound action potential amplitude and shape (5).

This group developed a closed loop spinal cord system that was used in this study. This system records the evoked compound action potential and uses this information in a feedback control mechanism (1). The measured evoked compound action potential amplitude is compared almost instantaneously to a reference, i.e., a "comfortable patient determined target level that provides optimal pain relief". This informs the newly calculated stimulus amplitude and the resultant current output. The current is adjusted continuously to create a constant evoked compound action potential amplitude (1). The goal is to maintain an electrical stimulation dose within therapeutic perception and discomfort thresholds, independent of the patient's involvement.

This 6-month study was a prospective, multi-center, single-arm study without a control group. Patients selected represented those similar to those seen in clinical practice, e.g., failure of conservative therapy for more than three month and diagnoses such as failed back surgery syndrome and radiculopathy. Pain, pain impact, function, sleep, and quality of life were surveyed at pre-specified time points out to 6 months. There were reductions in mean back and leg pain ratings at six months of $80.1 \%$ and $77.4 \%$ respectively. Notably, $>80 \%$ relief in back and leg pain (profound responders) at six months was achieved in $64.3 \%$ and $60.9 \%$ of patients respectively. Secondary outcomes demonstrated improvements in pain impact, function, sleep and quality of life. Opioid dose reduction was not evaluated in this study, but was evaluated in their follow-up twelve-month study (4). In terms of safety, two patients (3.9\%) developed serious adverse events requiring explant. These were not infectious complications or complications related to device malfunction. Other reported complications include noninfectious surgical site problems afflicting six patients $(11.8 \%)$ and lead migration afflicting 4 patients $(7.8 \%)$.

Overall, this is a well-designed study addressing an unmet need in tonic and low frequency spinal cord systems.
Firstly, selected patients represent a typical clinical sample in most pain practices. Secondly, experienced spinal cord systems implanters would not have to modify their approach or acquire new skills for implantation. Most importantly, this closed loop system harkens the proverb 'don't throw the baby out with the bathwater' with respect to tonic stimulation. Recent advances in spinal cord stimulation have focused on the type of electrical dose administration, i.e., waveform, to address long term patient dissatisfaction. There has been a movement away from low frequency or perceptible stimulation to high frequency and imperceptible stimulation. High frequency and burst stimulation are two advances in this sphere $(7,8)$. These latter advances allow anatomic placement of leads as opposed to physiological mapping with paresthesias (7). They avoid problems related to postural, heart rate, and respiratory variation in stimulation. However, the closed loop system addresses these limitations with conventional low frequency spinal cord systems by an evoked compound action potential sensing feedback loop.

These authors have further demonstrated that only a handful of fibers are recruited during spinal cord stimulation and generally the effect is localized to a few segments of the spinal cord. In their sheep study, the authors concluded that 'In reality, the majority of fibers recruited by SCS terminate in the cord within a few spinal segments". The relationship between the identity of recruited neurons and the degree of pain relief is unknown. However, it can be hypothesized that the recruitment of the locally terminating fibers is primarily responsible for spinal cord systems pain relief, while the recruitment of the long ascending fibers probably drives paresthesia perception and is only weakly related to the level of pain relief' (8). Hence, both low frequency and high frequency stimulation may be a localized phenomenon with differential activation of large versus small diameter fibers. The closed loop system may have been the missing link in optimizing low frequency systems so current delivery and activation stay within a therapeutic range most of the time. Their clinical trial demonstrates success pain, pain impact, function, sleep, and quality of life parameters. One major advance in this paper is the ability of low frequency stimulation to treat axial low back pain. The convergence of low frequency, high frequency, and burst stimulation in treating low back pain, coupled with neurophysiologic evidence of a localized effect over a few spinal cord segments and with the recruitment of merely a few small fibers suggests a bright future in understanding the mechanisms of spinal cord systems pain relief. 


\section{Acknowledgments}

Funding: None.

\section{Footnote}

Conflicts of Interest: All authors have completed the ICMJE uniform disclosure form (available at http://dx.doi. org/10.21037/apm-20-642). ADK reports personal fees from Merck, outside the submitted work; RDU reports grants from Medtronic, grants from Merck, outside the submitted work; RVS has nothing to disclose.

Ethical Statement: The authors are accountable for all aspects of the work in ensuring that questions related to the accuracy or integrity of any part of the work are appropriately investigated and resolved.

Open Access Statement: This is an Open Access article distributed in accordance with the Creative Commons Attribution-NonCommercial-NoDerivs 4.0 International License (CC BY-NC-ND 4.0), which permits the noncommercial replication and distribution of the article with the strict proviso that no changes or edits are made and the original work is properly cited (including links to both the formal publication through the relevant DOI and the license). See: https://creativecommons.org/licenses/by-nc-nd/4.0/.

\section{References}

1. Russo M, Cousins MJ, Brooker C, et al. Effective Relief of
Pain and Associated Symptoms With Closed-Loop Spinal Cord Stimulation System: Preliminary Results of the Avalon Study. Neuromodulation 2018;21:38-47.

2. Shah RV, Ericksen JJ, Lacerte M. Interventions in chronic pain management 2. New frontiers: invasive nonsurgical interventions. Arch Phys Med Rehabil 2003;84:S39-44.

3. Hofmeister M, Memedovich A, Brown S, et al. Effectiveness of Neurostimulation Technologies for the Management of Chronic Pain: A Systematic Review. Neuromodulation 2020;23:150-7.

4. Russo M, Brooker C, Cousins MJ, et al. Sustained Long-Term Outcomes With Closed-Loop Spinal Cord Stimulation: 12-Month Results of the Prospective, Multicenter, Open-Label Avalon Study. Neurosurgery 2020. [Epub ahead of print].

5. Parker JL, Karantonis DM, Single PS, et al. Compound action potentials recorded in the human spinal cord during neurostimulation for pain relief. Pain 2012;153:593-601.

6. Parker JL, Obradovic M, Hesam Shariati N, et al. Evoked Compound Action Potentials Reveal Spinal Cord Dorsal Column Neuroanatomy. Neuromodulation 2020;23:82-95.

7. Kirketeig T, Schultheis C, Zuidema X, et al. Burst Spinal Cord Stimulation: A Clinical Review. Pain Med 2019;20:S31-40.

8. Huang D, Song L, Li Y, et al. Posteromedial quadratus lumborum block versus transversus abdominal plane block for postoperative analgesia following laparoscopic colorectal surgery: A randomized controlled trial. J Clin Anesth 2020;62:109716.
Cite this article as: Shah RV, Kaye AD, Urman RD. Emerging concepts in the closed-loop spinal cord stimulation system: preliminary results of the Avalon study. Ann Palliat Med 2020;9(3):1272-1274. doi: 10.21037/apm-20-642 